\begin{tabular}{|c|l|}
\hline Title & Depth profiling analysis of solar wind helium collected in diamond-like carbon film from Genesis \\
\hline Author(s) & $\begin{array}{l}\text { Bajo, Ken-ichi; Olinger, Chad T.; Jurewicz, A my J. G.; Burnett, Donald S.; Sakaguchi, Isao; Suzuki, Taku; Itose, } \\
\text { Satoru; Ishihara, Morio; Uchino, Kiichiro; Wieler, Rainer; Y urimoto, Hisay oshi }\end{array}$ \\
\hline Citation & $\begin{array}{l}\text { Geochemical Journal, 49(5), 559-566 } \\
\text { https://doi.org/40.2343//3eochemj.2.0385 }\end{array}$ \\
\hline Issue Date & 2015 \\
\hline Doc URL & http://hdl.handle.net/2115/59846 \\
\hline Type & article \\
\hline File Information & GJ_49_p559.pdf \\
\hline
\end{tabular}

Instructions for use 


\title{
Depth profiling analysis of solar wind helium collected in diamond-like carbon film from Genesis
}

\author{
Ken-IChi Bajo, ${ }^{1}$ Chad T. Olinger, ${ }^{2}$ Amy J. G. Jurewicz, ${ }^{3}$ Donald S. Burnett, ${ }^{4}$ Isao SAKaguchi, ${ }^{5}$ TAKu SuZuKi, ${ }^{5}$

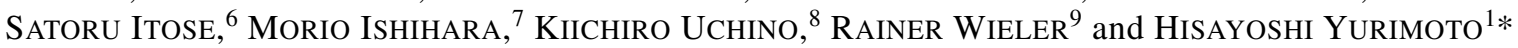 \\ ${ }^{1}$ Department of Natural History Sciences, Hokkaido University, IIL, Sapporo 001-0021, Japan \\ ${ }^{2}$ Applied Modern Physics Group, Los Alamos National Laboratory, Los Alamos, NM 87545, U.S.A. \\ ${ }^{3}$ CMS/SESE, Arizona State University, Tempe, AZ 85287-1404, U.S.A. \\ ${ }^{4}$ Division of Geological and Planetary Sciences, California Institute of Technology, Pasadena, CA 91125, U.S.A. \\ ${ }^{5}$ National Institute for Materials Science, Tsukuba, Ibaraki 305-0044, Japan \\ ${ }^{6}$ JEOL Ltd., Musashino, Akishima, Tokyo 196-8558, Japan \\ ${ }^{7}$ Department of Physics, Osaka University, Toyonaka, Osaka 560-0043, Japan \\ ${ }^{8}$ Graduate School of Engineering Sciences, Kyushu University, Kasuga, Fukuoka 816-8580, Japan \\ ${ }^{9}$ Institute for Isotope Geology and Mineral Resources, ETH Zurich, Clausiusstrasse 25, 8092 Zurich, Switzerland
}

(Received January 23, 2015; Accepted June 30, 2015)

\begin{abstract}
The distribution of solar-wind ions in Genesis mission collectors, as determined by depth profiling analysis, constrains the physics of ion-solid interactions involving the solar wind. Thus, they provide an experimental basis for revealing ancient solar activities represented by solar-wind implants in natural samples. We measured the first depth profile of ${ }^{4} \mathrm{He}$ in a Genesis collector; the shallow implantation (peaking at $<20 \mathrm{~nm}$ ) required us to use sputtered neutral mass spectrometry with post-photoionization by a strong field. The solar wind He fluence calculated using depth profiling is $\sim 8.5 \times 10^{14} \mathrm{~cm}^{-2}$. The shape of the solar wind ${ }^{4} \mathrm{He}$ depth profile is consistent with TRIM simulations using the observed ${ }^{4} \mathrm{He}$ velocity distribution during the Genesis mission. It is therefore likely that all solar-wind elements heavier than $\mathrm{H}$ are completely intact in this Genesis collector and, consequently, the solar particle energy distributions for each element can be calculated from their depth profiles. Ancient solar activities and space weathering of solar system objects could be quantitatively reproduced by solar particle implantation profiles.
\end{abstract}

Keywords: solar wind, depth profile, helium, NASA Genesis mission, post-ionization

\section{INTRODUCTION}

NASA's Genesis mission collected samples of solar wind that can be analyzed with high precision in laboratories with the ultimate goal of determining the composition of the sun and the solar nebula from which it was formed (Burnett, 2013). The solar wind was implanted in Genesis collector materials, which were various ultrahighpurity materials specifically chosen for laboratory analyses (Jurewicz et al., 2003). Understanding the details of solar-wind activity requires time-dependent characterization of solar wind conditions and general compositional changes during the collection periods. This characterization has been done in-situ using the Ion Monitor (GIM) on Genesis and the Solar-Wind Ion Composition Spectrometer (SWICS) on the Advanced Composition Explorer (ACE) (Reisenfeld et al., 2013). Therefore, the

*Corresponding author (e-mail: yuri@ep.sci.hokudai.ac.jp)

Copyright @ 2015 by The Geochemical Society of Japan. physical conditions for ion implantation of solar wind elements in Genesis collectors are well defined. In particular, the distribution of energies at which the solar-wind ions were implanted is known.

A Monte Carlo program, the Transport of Ions in Matter (TRIM), is useful for analyzing ion implantation profiles (Ziegler et al., 2012). However, TRIM simulations may overestimate the projected range of ions for low-energy ion implantation (less than several kiloelectron volts) (Nakajima et al., 2001). Therefore, implantation profiles for low-energy solar wind elements (e.g., $\mathrm{H}$ and $\mathrm{He}$ ) should be carefully evaluated experimentally using laboratory ion implantation. In this context, depth-profiles from analyses of Genesis collectors document the physics of ion-solid interactions caused by the solar wind that were previously only modeled by TRIM and provide an experimental basis for revealing ancient solar activities from solar wind implants in natural samples (from the moon, asteroids, and so on).

Helium is the second most abundant element in the solar wind ( 4\% atomic abundance) and a minor element 

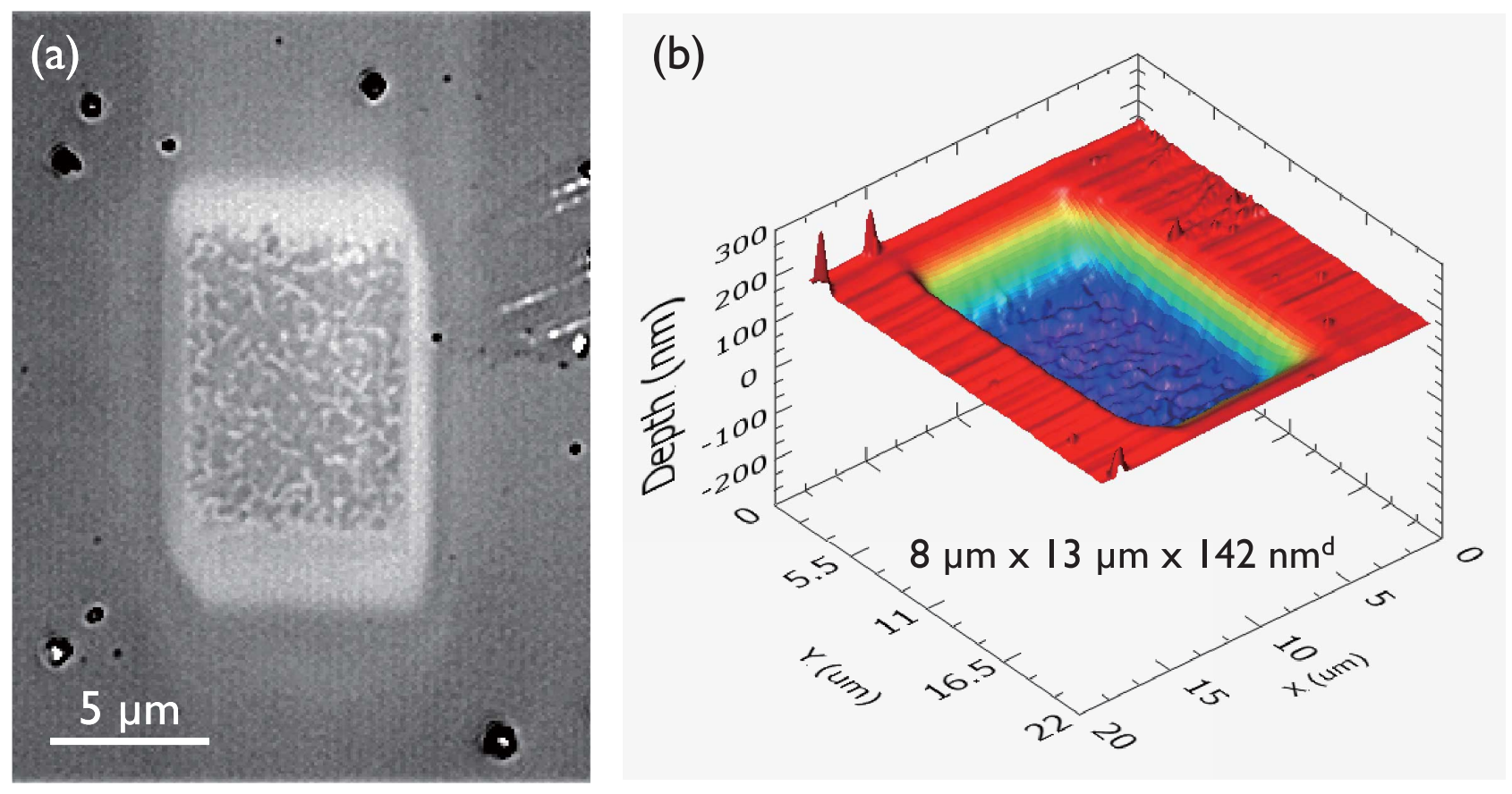

Fig. 1. Sputter crater on Genesis DOS sample. (a) Laser scanning confocal microscope image. (b) 3-D image obtained by atomic force microscope.

in the terrestrial atmosphere ( $\sim 5 \mathrm{ppm}$ atomic). Thus, we can expect that (1) abundant $\mathrm{He}$ is implanted in Genesis collectors, and (2) the He implantation profiles observed do not include terrestrial contamination. Indeed, large quantities of solar wind He implanted in Genesis collectors $\left(\sim 10^{15}\right.$ atoms $\left.\mathrm{cm}^{-2}\right)$ have been measured by conventional laser ablation mass spectrometry essentially without terrestrial contamination (Heber et al., 2009, 2012). However, the solar wind implantation profiles were not measured because conventional depth-profiling methods (e.g., secondary ion mass spectrometry) give extremely low ionization yields for He (Gnaser and Oechsner, 1991; Warhaut et al., 1979).

In this study, we used a novel technique-sputtered neutral mass spectrometry (SNMS) using strong-field ionization* (Ebata et al., 2012)-to measure He profiles in Genesis collectors, thus revealing the nanoscale depth distribution of solar wind He. We can obtain depth profiles because our instrument can quantify ${ }^{4} \mathrm{He}$ present at tens of parts per millions atomic by analyzing an area of a solid surface only a few microns in size. Here we report

*Strong-field ionization is a process in which electrons in an atom (or a molecule) pass through the potential barrier by tunneling. The potential barrier of an atom (molecule) is drastically distorted in an intense electric field caused by a femtosecond laser pulse. Therefore, electrons can escape from the atom (molecule).

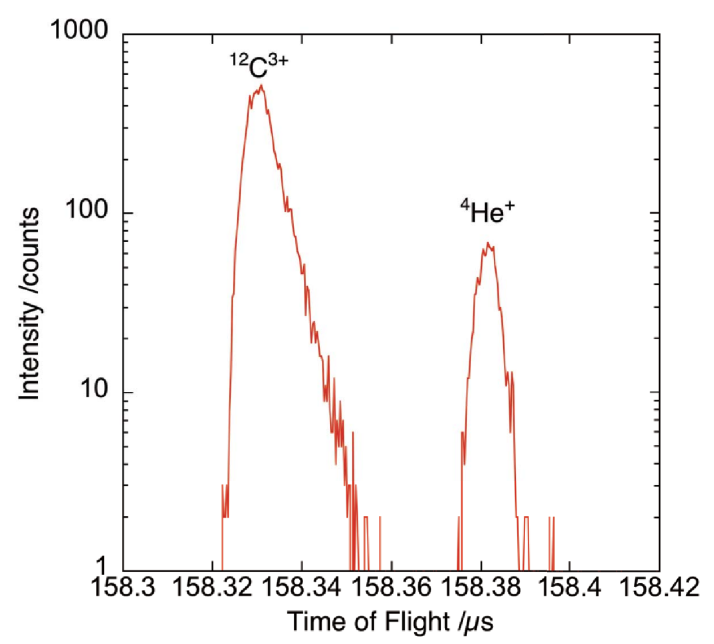

Fig. 2. Mass spectrum at $m / z=4$ from Genesis DOS sample.

the first results for a depth distribution profile of solar wind ${ }^{4} \mathrm{He}$ from a Genesis collector, a diamond-like carbon film on a silicon (DOS) wafer. The solar wind ${ }^{4} \mathrm{He}$ distribution is compared with the results of laboratory experiments of ${ }^{4} \mathrm{He}$ ion implantation and TRIM simulations. We also discuss solar wind implantation in natural samples and the implications for space and solar physics. 

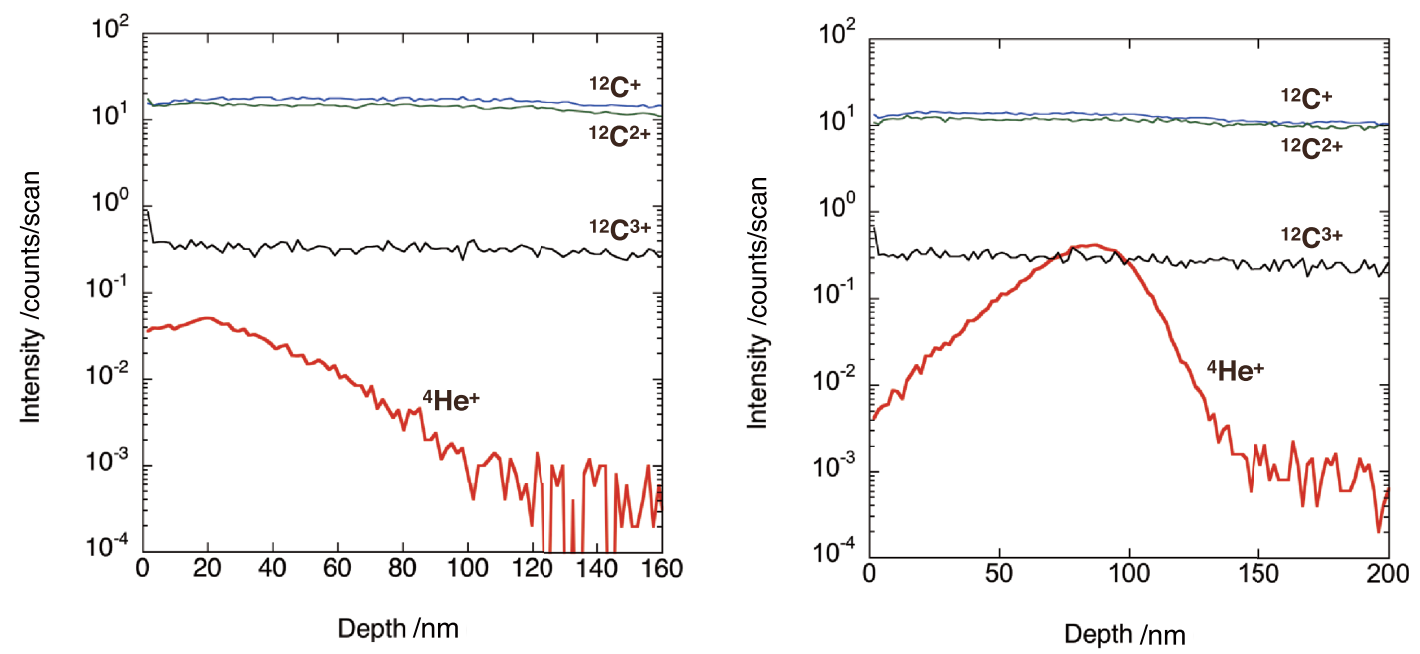

Fig. 3. Depth profile of ion intensity on 2014/3/11. (a) Genesis DOS sample. (b) Ion-implanted DOS sample.

\section{EXPERIMENTAL}

A diamond-like-carbon (DLC) film deposited with a thickness of $1.5-3 \mu \mathrm{m}$ on a silicon wafer substrate (DOS 60939) from the Genesis B/C arrays was used in this study (Jurewicz et al., 2003). The B/C arrays collected bulk solar wind and were exposed continuously during the science collection period of Genesis, which totaled 852.83 days (Reisenfeld et al., 2013). Non-flight DOS samples, both without and with commercial He ion implants, were also used to determine our detection limit and to determine concentrations for implanted solar wind, respectively. The experiments on commercially implanted non-flight DOS samples contained ${ }^{4} \mathrm{He}^{+}$implanted at an energy of $15 \mathrm{keV}$ and having a nominal fluence of $1 \times 10^{16} \mathrm{~cm}^{-2}$ (Leonard Kroko, Inc.).

An SNMS instrument called LIMAS (Ebata et al., 2012) was used for our measurements. A pulsed primary beam of ${ }^{69} \mathrm{Ga}$ (30 keV and $38 \mathrm{nA}$ ) was focused to a spot $800 \mathrm{~nm}$ in diameter on the sample surface of the DOS. The pulse period was set to $500 \mathrm{~ns}$. Sputtered neutrals were ionized by a focused ( $50 \mu \mathrm{m}$ diameter) femtosecond laser beam under a strong-field ionization condition. The laser pulse was $40 \mathrm{fs}$ with an energy of $2.5 \mathrm{~mJ}$ and $1 \mathrm{kHz}$ repetition. We accumulated post-ionized ions for 200 primary beam pulses at each spot to collect data to discriminate $\mathrm{He}$ depth distributions. The primary beam was rastered to form a crater on the sample over a pattern of $15 \times 15$ spots with a step interval of $500 \mathrm{~nm}$ (Fig. 1). As a result, the raster covered an area of $8 \times 13 \mu \mathrm{m}^{2}$ on the surface because the incident angle of the primary beam, formed by the optical axis of the primary beam on the surface and the normal to the surface at the point of incidence, was set to $55^{\circ}$. At this angle, the DOS surface be- came perpendicular to the optical axis of the ion extraction optics into the mass spectrometer.

Positive ions were introduced into a multi-turn timeof-flight mass spectrometer (Okumura et al., 2004) by an acceleration voltage of $-4 \mathrm{kV}$ with $1 \mathrm{kHz}$ repetition (synchronized with the primary ion pulses). Measurements were made at all the rastered primary beam spots, and only ions generated from the center $\left(2.5 \times 4 \mu \mathrm{m}^{2}, 5 \times 5\right.$ spots) of the sputtered craters were used to determine the depth profiles in order to avoid crater edge effects. The flight path length for the mass spectrometer was set to 68 $\mathrm{m}$ for ${ }^{4} \mathrm{He}^{+}$. We measured ${ }^{4} \mathrm{He}^{+},{ }^{12} \mathrm{C}^{3+},{ }^{12} \mathrm{C}^{2+}$, and ${ }^{12} \mathrm{C}^{+}$. The integral intensities of each peak were used for their ion intensities. The ${ }^{4} \mathrm{He}^{+}$intensities were normalized to the total intensities of ${ }^{12} \mathrm{C}^{3+},{ }^{12} \mathrm{C}^{2+}$, and ${ }^{12} \mathrm{C}^{+}$and translated to concentrations using a calibration factor (relative sensitivity factor) obtained from the ion-implanted DOS sample with reference to International Standard ISO 18114:2003 (Simons, 2006). The vacuum of the sample chamber was maintained at $3 \times 10^{-8} \mathrm{~Pa}$ during measurements. The depths of sputtered craters were measured after depth profiling analyses by an atomic force microscope (Asylum Technology, MFP-3D-BIO-J).

\section{RESULTS AND DISCUSSION}

Two peaks at $m / z=4$ are observed from Genesis DOS 60939, corresponding to ${ }^{4} \mathrm{He}^{+}$and ${ }^{12} \mathrm{C}^{3+}$ (Fig. 2). The mass resolution is calculated to be $M / \Delta M=8600$ (at $10 \%$ valley) with a peak width of $5 \mathrm{~ns}$ (FWHM). The ${ }^{4} \mathrm{He}$ peak has no interference under these conditions.

Depth profiles from DOS 60939 and an ion-implanted DOS reference are shown in Fig. 3. The intensities of carbon ions appear constant during analysis, indicating a 


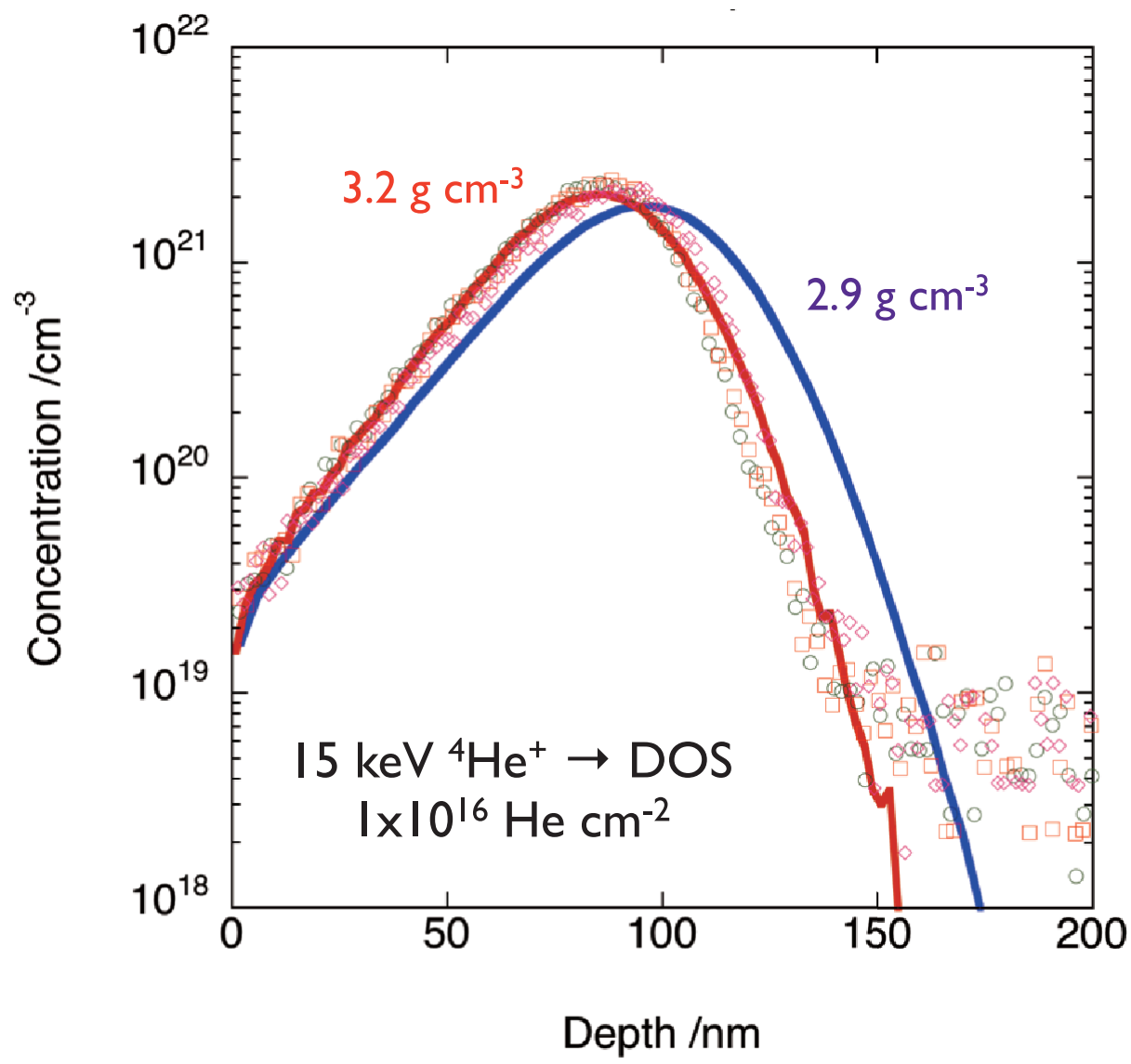

Fig. 4. Depth profile of ${ }^{4}$ He concentration implanted in DOS. Three independent measurements plotted as circles (2014/3/11), squares (2014/3/12), and diamonds (2014/3/14) are shown. Distributions calculated by TRIM simulation are shown by lines. Data are shown in Table S1.

stable sputtering rate and stable post-ionization. Therefore, we assume a constant sputter rate when converting time to depth for each measurement and adopt an average sputtering rate determined by dividing the total crater depth by the total sputter time. The ${ }^{4} \mathrm{He}$ profiles strongly suggest an ion implant, but the background $(\sim 3$ $\times 10^{-4}$ counts/scan) is high. The background is also observed on the DOS sample without ion implantation from the surface to $\sim 30 \mathrm{~nm}$ in depth under the same measurement condition and shows $2-3 \times 10^{-4}$ counts/scan as a blank. The blank signals represent ${ }^{4} \mathrm{He}$ on the basis of their position in the mass spectrum. The similar intensities for all samples indicate that the ${ }^{4} \mathrm{He}$ blank is controlled by ${ }^{4} \mathrm{He}$ gas in the photoionization volume on the sample surface. The He gas comes from residual $\mathrm{He}$ in vacuum and adsorbed $\mathrm{He}$ on the surface sputtered by the primary beam. Note that the sample chamber was maintained at ultrahigh vacuum $\left(3 \times 10^{-8} \mathrm{~Pa}\right)$.

The ion intensity ratios of ${ }^{4} \mathrm{He}^{+} /\left(\mathrm{C}^{+}+\mathrm{C}^{2+}+\mathrm{C}^{3+}\right)$ from the ion-implanted DOS sample are converted to concentrations $\left(\right.$ atoms $\mathrm{cm}^{-3}$ ) by normalization to the nominal ion fluence, which is realized by setting the integral of the measured depth distribution equal to the implanted fluence (Fig. 4, Supplementary Table S1). The distribution of ${ }^{4} \mathrm{He}$ may also be simulated using the TRIM program. The results of the TRIM simulation are sensitive to both the chemical bonding strength and the density of materials, in addition to the composition of the substrate (Ziegler $e t$ al., 2012). The DLC film is usually characterized as amorphous with a density of $2.9 \mathrm{~g} \mathrm{~cm}^{-3}$ and diamond-like chemical bonding (unpublished laboratory data of AJGJ). However, a ${ }^{4} \mathrm{He}$ depth-distribution calculated by TRIM using these parameters overestimates the projected range of ${ }^{4} \mathrm{He}$ ions into DLC in comparison with the observed depth profile of the implanted ${ }^{4} \mathrm{He}$ ions. On the other hand, a TRIM calculation assuming a density of $3.2 \mathrm{~g} \mathrm{~cm}^{-3}$ better simulates the observed projected range, peak concentration, and overall distribution of ${ }^{4} \mathrm{He}$ ions. This discrepancy may indicate an inhomogeneity in density within the amorphous DLC or may reflect some other variable in the material that mimics the effect of higher density. In any case, the success of the simulation assuming a den- 


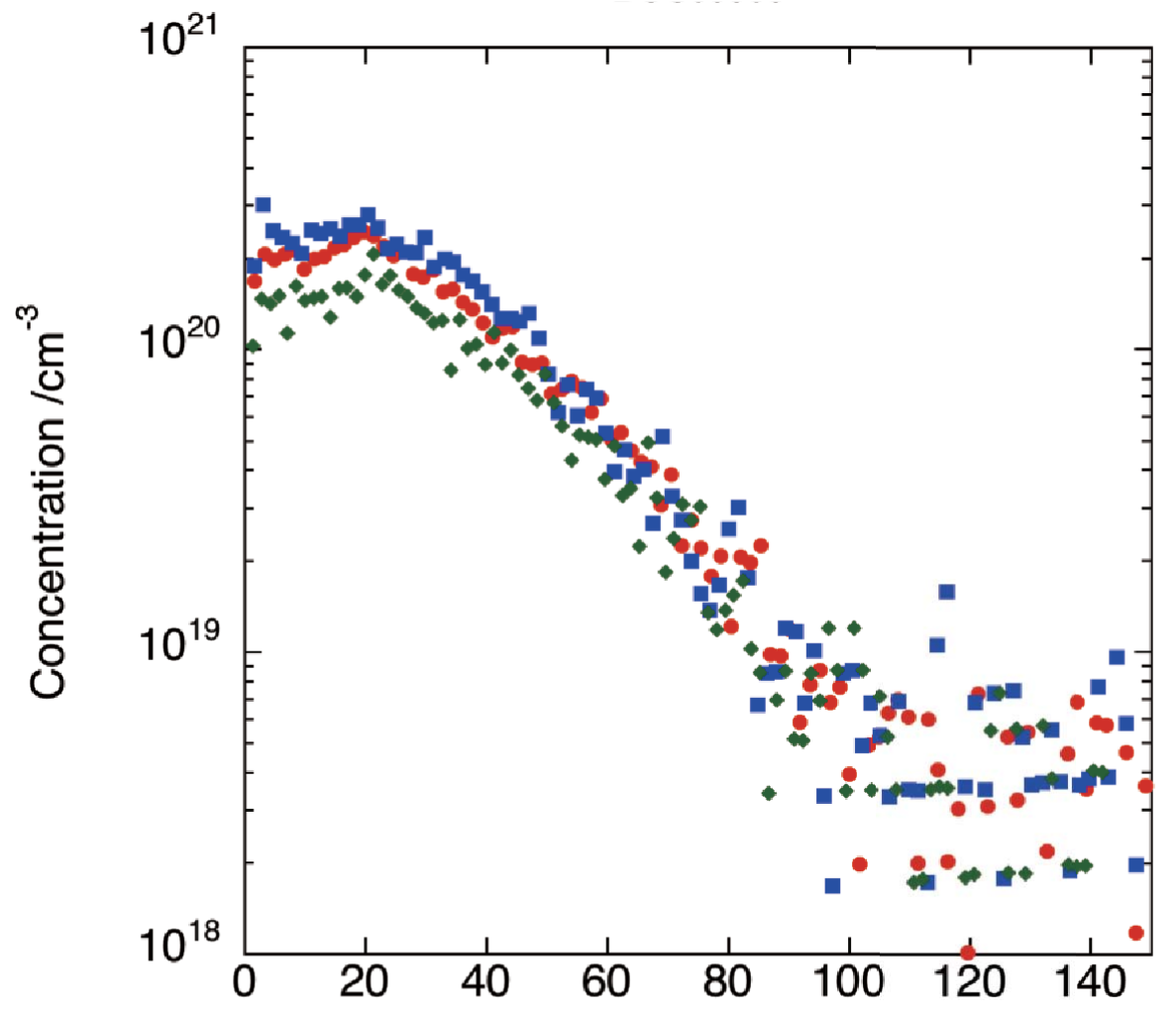

\section{Depth /nm}

Fig. 5. Depth profile of solar wind ${ }^{4}$ He concentration from Genesis DOS sample. Three independent measurements plotted as circles (2014/3/11), squares (2014/3/12), and diamonds (2014/3/14) are shown.

sity of $3.2 \mathrm{~g} \mathrm{~cm}^{-3}$ indicates that incident ion energies can be calculated from a measured depth distribution if we adopt an appropriate density parameter for the TRIM simulation. From these observations, the useful yields, (numbers of ions detected)/(total numbers of atoms sputtered), of $\mathrm{C}$ and $\mathrm{He}$ are calculated to be $\sim 0.00007$ and $\sim 0.00004$, respectively, under this measurement condition.

The concentrations of solar wind He from the Genesis DOS 60939 sample are calculated from the ion intensities using the calibration factor (relative sensitivity factors shown in Table S1) determined by the ion-implanted DOS measurement conducted on the same day under exactly the same measurement condition. We conducted DOS 60939 measurements on three different days and calculated He concentrations using the calibration factor for the day. Figure 5 shows three independent measurements of the solar wind ${ }^{4} \mathrm{He}$ distribution from the DOS 60939 sample. The ${ }^{4} \mathrm{He}$ distributions are independent measurements and yet are parallel to each other, which indicates that the relative depth distribution is reproducible. The absolute concentration at a given depth, how- ever, is variable for each measurement because calibration factors from ion intensities to ${ }^{4} \mathrm{He}$ concentrations may vary with the measurement area. That is, because He has a much lower ionization efficiency by laser $(\sim 10 \%)$ than all other elements $(\sim 100 \%)$ (Ebata et al., 2012), the ionization efficiency of ${ }^{4} \mathrm{He}$ strongly depends on the laser photon density, i.e., the focusing conditions at the postionization position. Therefore, the extraction efficiency of each post-ionized ion for LIMAS would vary from spot to spot because the geometric relationship between the sputtering and the laser focusing changes slightly each time the sample moves. Nevertheless, the variation in the measured concentration between independent measurements did not exceed a factor of two (Fig. 5). The calculated ${ }^{4} \mathrm{He}$ fluences for each measurement are 6.7, 8.7, and $9.7 \times 10^{14} \mathrm{~cm}^{-2}$, respectively. The recommended ${ }^{4} \mathrm{He}$ fluence of solar wind during the Genesis mission in this study is calculated by the averaged profile shown in Fig. 6 and found to be $8.5 \times 10^{14} \mathrm{~cm}^{-2}$ with a standard deviation of the mean of $0.7 \times 10^{14}$, consistent with the accumulated value during solar-wind irradiation periods monitored by the GIM $\left(8.09 \times 10^{14} \mathrm{~cm}^{-2}\right)$ and SWICS/ACE 


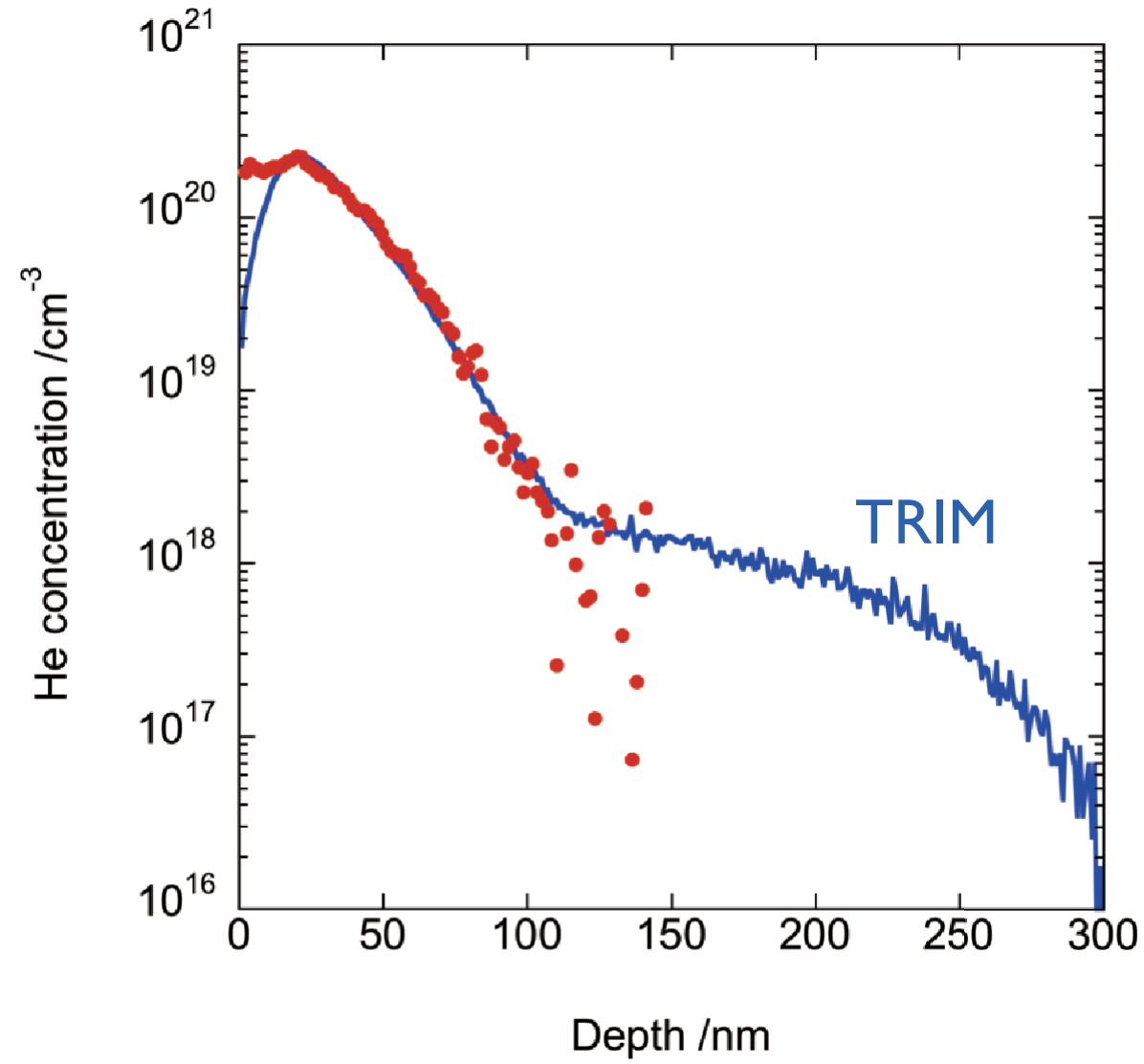

Fig. 6. Average depth profile of solar wind ${ }^{4} \mathrm{He}$ into Genesis DOS sample. Circles are from observation with background correction. Line is calculated by TRIM simulation assuming density of $3.2 \mathrm{~g} \mathrm{~cm}^{-3}$ using energy distributions of solar wind ${ }^{4} \mathrm{He}$ observed by ACE/SWICS (Reisenfeld et al., 2013).

$\left(8.58 \times 10^{14} \mathrm{~cm}^{-2}\right)$ in situ (Reisenfeld et al., 2013), as well as in DOS by laser ablation sample analysis $(8.29 \times$ $10^{14} \mathrm{~cm}^{-2}$ ) (Heber et al., 2009).

The averaged depth profile after background subtraction is shown in Fig. 6. The average profile is calculated by depth interpretation from data shown in Fig. 5. We use the background signals of the day for the subtractions. The distribution has a peak at $\sim 20 \mathrm{~nm}$, and the peak concentration is about $2.2 \times 10^{20} \mathrm{~cm}^{-3}$. The distribution decreases with depth following an approximately Gaussian shape. A similar decrease is also observed from the maximum toward the surface before the distribution becomes flat at depths less than $\sim 15 \mathrm{~nm}$.

We simulated the implant distribution of solar wind ${ }^{4} \mathrm{He}$ collected during the Genesis mission by TRIM (Fig. 6) using data based on the solar wind energy distributions from ACE/SWICS (Reisenfeld et al., 2013). In the simulation, we assumed that the density of the DLC film is $3.2 \mathrm{~g} \mathrm{~cm}^{-3}$ according to the discussion for the ion-implanted DOS sample. The simulated distribution reproduces the projected range, the deep portion of the profile, and the absolute concentration at a given depth except for the topmost $15 \mathrm{~nm}$, where the measured profile is almost flat (Fig. 6). A long tail is predicted in the TRIM simulation, which is almost equivalent to the detection limits for the blank.

Figure 1 shows a sputter crater on the DOS 60939 sample. The roughness of the DOS surface and the crater bottom are typically $1.4 \mathrm{~nm}(\mathrm{rms})$ and $4.2 \mathrm{~nm}$ (rms), respectively, suggesting that degradation of depth resolution is small during each analysis. On the other hand, sputtering by $30 \mathrm{keV} \mathrm{Ga}$ ions leads to atom mixing in a layer 10-20 $\mathrm{nm}$ thick because atomic mixing effects occur within a layer corresponding to the projected range, which is calculated to be $\sim 15 \mathrm{~nm}$ into the DOS substrate for $30 \mathrm{keV}$ Ga ions by TRIM. Thus, the depth resolution in this study would be limited to $10-20 \mathrm{~nm}$. This is indeed consistent with the thickness of the surface layer in which the data deviate from the model predictions. This demonstrates that solar wind $\mathrm{He}$ is quantitatively retained in Genesis collectors and that its depth distribution accurately preserves the original distribution upon implantation in the 
Genesis collector. All elements heavier than He are probably also quantitatively retained in the amorphous DLC film collector because, typically, their mobility in solids is slower than that of He. Consequently, the solar-wind energy distribution can be quantitatively derived from the implantation distribution of the solar wind if the density parameter of the TRIM simulation is appropriate.

Surfaces of extraterrestrial natural samples directly irradiated by solar wind are covered by a deposition layer tens of nanometers in thickness (Keller and McKay, 1993; Noguchi et al., 2014). On the other hand, deposition layers have not been observed in Genesis samples (Calaway et al., 2009), although an organic film contamination layer ("brown stain") has been observed on some Genesis samples. The deposition layer must have grown slowly over geological time. Irradiation of solar wind would have continued during growth of the deposition layer. Therefore, depth profiles of ${ }^{4} \mathrm{He}$ in extraterrestrial samples will be the result of simultaneous deposition of material and implantation/sputtering of solar wind ions. Indeed, depth profiles in extraterrestrial samples would differ from those in the Genesis samples. From the difference, we may calculate the solar wind exposure time of the extraterrestrial samples corresponding to the speed of space weathering.

Solar-type stars have recently been observed to generate superflares more frequently than previously thought (Maehara et al., 2012). Superflares are accompanied by coronal mass ejections (CMEs), and the energies are more than thousands of times larger than those in present solar flares on the sun. Superflares have not been observed from the sun so far; they would occur at a frequency of several times in ten thousand years if they occur at all, but meteoritic or lunar materials may record these events in their He depth distributions. LIMAS has the potential to detect superflares of the ancient sun and to infer their frequency and energies from extraterrestrial samples.

The tail of the TRIM simulation curve deeper than $120 \mathrm{~nm}$ (Fig. 6) corresponds to CME solar wind. At these depths, the background level in this study is close to the predicted concentration in Genesis E array collectors induced by the October 2003 CME during the Genesis mission. CME He in Genesis samples could be detected if the partial pressure of the He in the sample chamber of LIMAS is decreased, because the background is limited by residual He gas in vacuum. The residual He gas would be the result of penetration through glass viewports on the LIMAS sample chamber. We are planning to remodel the glass viewports on the LIMAS sample chamber to reduce He influx from the air.

\section{SUMMARY}

NASA's Genesis solar wind sample return mission is a unique experiment that allows us to probe the physics of ion-solid interaction caused by the solar wind and will provide an experimental basis for the analysis of natural samples irradiated by the solar wind. For the first time, depth profiling of solar wind ${ }^{4} \mathrm{He}$ has been done on a Genesis DOS sample; SNMS with post-photoionization by a strong field was used. The ion intensities of the postionized isotopes were converted to concentrations by comparison with ion intensities from a DOS standard that was implanted with ${ }^{4} \mathrm{He}^{+}$ions at $15 \mathrm{keV}$.

The depth profile represents a measured layer $140 \mathrm{~nm}$ in depth and $2.5 \times 4 \mu \mathrm{m}^{2}$ in area. The peak concentration of implanted solar wind ${ }^{4} \mathrm{He}$ is about $2.2 \times 10^{20} \mathrm{~cm}^{-3}$ at $\sim 20 \mathrm{~nm}$ in depth. The implantation profile is traced to $100 \mathrm{~nm}$ in depth until the blank level is reached, which results from ${ }^{4} \mathrm{He}$ due to photoionization of residual ${ }^{4} \mathrm{He}$ gas in vacuum and sputtered ${ }^{4} \mathrm{He}$ adsorbed on the surface from vacuum during measurements. The blank corresponds to $\sim 3 \times 10^{18} \mathrm{~cm}^{-3}$. The solar wind ${ }^{4} \mathrm{He}$ fluence calculated by the depth-profiling method $\left(\sim 8.5 \times 10^{14}\right.$ $\mathrm{cm}^{-2}$ ) is consistent with those determined by previous laboratory measurements (Heber et al., 2009).

The solar wind ${ }^{4} \mathrm{He}$ distribution in the DOS sample was compared with a distribution calculated by TRIM using the solar wind energy distribution during the Genesis mission from ACE/SWICS. The projected range, peak concentration, and concentration at a given depth are simulated by TRIM if an appropriate density parameter is adopted. Assuming He has the highest mobility among all elements other than $\mathrm{H}$, this analysis demonstrates that all solar-wind elements heavier than $\mathrm{H}$ are completely intact in this Genesis collector material. Consequently, the solar-wind energy distribution can, ideally, be calculated from measured depth profiles, and the profiles can be useful for understanding ancient solar activities experienced by natural samples, as well as space weathering evolution of solar system objects.

Acknowledgments - We thank Hajime Hiyagon and an anonymous reviewer for their constructive reviews and suggestions and Yuji Sano for his kind editorial advice. This study is supported in part by Monka-sho grants. This is a contribution to Los Alamos publication LA-UR-15-21508.

\section{REFERENCES}

Burnett, D. S. (2013) The Genesis solar wind sample return mission: Past, present, and future. Meteorit. Planet. Sci.48, 2351-2370.

Calaway, M. J., Stansbery, E. K. and Keller, L. P. (2009) Genesis capturing the sun: Solar wind irradiation at Lagrange 1. Nucl. Instrum. Methods Phys. Res., Sect. B 267, 11011108.

Ebata, S., Ishihara, M., Uchino, K., Itose, S., Matsuya, M., Kudo, M., Bajo, K.-i. and Yurimoto, H. (2012) Development of laser ionization mass nanoscope (LIMAS). Surf. Interface Anal. 44, 635-640. 
Gnaser, H. and Oechsner, H. (1991) Noval detection scheme for the analysis of hydrogen and helium by secondary ion mass spectrometry. Surf. Interface Anal. 17, 646-649.

Heber, V. S., Wieler, R., Baur, H., Olinger, C., Friedmann, T. A. and Burnett, D. S. (2009) Noble gas composition of the solar wind as collected by the Genesis mission. Geochim. Cosmochim. Acta 73, 7414-7432.

Heber, V. S., Baur, H., Bochsler, P., McKeegan, K. D., Neugebauer, M., Reisenfeld, D. B., Wieler, R. and Wiens, R. C. (2012) Isotopic mass fractionation of solar wind: Evidence from fast and slow solar wind collected by the Genesis mission. Astrophys. J. 759, 121.

Jurewicz, A. J. G., Burnett, D. S., Wiens, R. C., Friedmann, T. A., Hays, C. C., Hohlfelder, R. J., Nishiizumi, K., Stone, J. A., Woolum, D. S., Becker, R., Butterworth, A. L., Campbell, A. J., Ebihara, M., Franchi, I. A., Heber, V., Hohenberg, C. M., Humayun, M., McKeegan, K. D., McNamara, K., Meshik, A., Pepin, R. O., Schlutter, D. and Wieler, R. (2003) The Genesis solar-wind collector materials. Space Sci. Rev. 105, 535-560.

Keller, L. P. and McKay, D. S. (1993) Discovery of vapor deposits in the lunar regolith. Science 261, 1305-1307.

Maehara, H., Shibayama, T., Notsu, S., Notsu, Y., Nagao, T., Kusaba, S., Honda, S., Nogami, D. and Shibata, K. (2012) Superflares on solar-type stars. Nature 485, 478-481.

Nakajima, K., Toyofuku, H. and Kimura, K. (2001) Anomalous surface amorphization of $\mathrm{Si}(001)$ induced by $3-5 \mathrm{keV}$ $\mathrm{Ar}^{+}$ion bombardment. Jpn. J. Appl. Phys. 40, 2119-2122.

Noguchi, T., Kimura, M., Hashimoto, T., Konno, M., Nakamura, T., Zolensky, M. E., Okazaki, R., Tanaka, M., Tsuchiyama, A., Nakato, A., Ogami, T., Ishida, H., Sagae, R., Tsujimoto, S., Matsumoto, T., Matsuno, J., Fujimura, A., Abe, M., Yada, T., Mukai, T., Ueno, M., Okada, T., Shirai, K. and Ishibashi,
Y. (2014) Space weathered rims found on the surfaces of the Itokawa dust particles. Meteorit. Planet. Sci. 49, 188214.

Okumura, D., Toyoda, M., Ishihara, M. and Katakuse, I. (2004) A compact sector-type multi-turn time-of-flight mass spectrometer 'MULTUM II'. Nucl. Instrum. Methods Phys. Res., Sect. A 519, 331-337.

Reisenfeld, D. B., Wiens, R. C., Barraclough, B. L., Steinberg, J. T., Neugebauer, M., Raines, J. and Zurbuchen, T. H. (2013) Solar wind conditions and composition during the Genesis mission as measured by in situ spacecraft. Space Sci. Rev. 175, 125-164.

Simons, D. S. (2006) Summary of ISO/TC 201 standard: XIII, ISO 18114:2003-surface chemical analysis—secondary ion mass spectrometry-determination of relative sensitivity factors from ion-implanted reference materials. Surf. Interface Anal. 38, 171-172.

Warhaut, M., Kiko, J. and Kirsten, T. (1979) Microdistribution patterns of implanted rare gases in a large number of individual lunar soil particles. Lunar and Planetary Science Conference, 10th, Houston, Tex., March 19-23, 1979, Proceedings, Volume 2, pp. 1531-1546, Pergamon Press, Inc., New York.

Ziegler, J. F., Biersack, J. P. and Ziegler, M. D. (2012) SRIM, The Stopping and Range of Ions in Matter. Lulu Press Co. (http://www.srim.org).

\section{SUPPLEMENTARY MATERIALS}

URL (http://www.terrapub.co.jp/journals/GJ/archives/ data/49/MS385.pdf)

Table S1 